\title{
EDITORIAL
}

\section{Histone deacetylases in RA: epigenetics and epiphenomena}

\author{
Aleksander M Grabiec and Kris A Reedquist* \\ See related research by Kawabata et al., http://arthritis-research.com/content/12/4/R133
}

\begin{abstract}
Reduced synovial expression of histone deacetylases (HDACs) is proposed to contribute to pathology in rheumatoid arthritis (RA) by enhancing histonedependent access of transcription factors to promoters of inflammatory genes. In the previous issue of Arthritis Research \& Therapy, Kawabata and colleagues provided independent evidence that HDAC activity is increased in the synovium and fibroblast-like synoviocytes (FLSs) of patients with RA and is paralleled by increased HDAC1 expression and synovial tumor necrosis factoralpha (TNFa) production. Remarkably, stimulation of RA FLSs with TNFa specifically increases HDAC activity and HDAC1 expression, suggesting that changes in synovial HDAC activity and expression may be secondary to local inflammatory status.
\end{abstract}

In the previous issue of Arthritis Research \& Therapy, Kawabata and colleagues [1] examined the activity and expression of histone deacetylases (HDACs) in the synovial tissue of patients with rheumatoid arthritis (RA) in relation to local tumor necrosis factor-alpha (TNFo) production. The authors found that total HDAC activity was increased in RA synovial tissue compared with osteoarthritis (OA) disease control and normal control tissues. Expression of HDAC1 was significantly elevated in RA among HDACs examined. Similarly, HDAC1 expression was elevated in RA fibroblast-like synoviocytes (FLSs) compared with OA FLSs. Both total synovial HDAC activity and HDAC1 expression were associated with increased TNF $\alpha$ production, and in attempting to understand the cellular basis of this relationship, the authors found that TNF $\alpha$ stimulation of RA FLSs led to

*Correspondence: k.a.reedquist@amc.uva.nl

Division of Clinical Immunology and Rheumatology, Academic Medical Center, University of Amsterdam, Meibergdreef 9, 1105 AZ Amsterdam, The Netherlands transient increases in cellular HDAC activity and HDAC1 expression. This report is noteworthy for the efforts of the authors to resolve apparent discrepancies between their data and the published literature [2] and for the resulting new questions regarding how HDACs might contribute to RA.

Histone acetyltransferases (HATs) and HDACs reciprocally regulate the acetylation status of cellular proteins. Acetylation of histones promotes unwinding of compacted chromatin and allows access of transcription factors to gene promoter regions, and by extension, changes in relative HAT/HDAC activity would be expected to influence the sensitivity of cellular gene transcription in response to extracellular stimuli. This epigenetic mechanism of gene regulation has been suggested to contribute to pathology in complex immune-mediated inflammatory diseases, such as chronic obstructive pulmonary disease (COPD) and asthma, in which depressed HDAC activity at the site of inflammation, especially in macrophages, is associated with disease severity and inflammatory cytokine production and contributes to glucocorticoid resistance [3]. An initial examination of HAT and HDAC activity in RA synovial tissue [2] painted a picture suggesting many similarities with COPD and asthma [4], noting depressed synovial HDAC activity in RA tissue compared with OA and normal donor tissues, particularly in regard to HDAC1 and HDAC2 expression. This initial study suggests that decreased HDAC activity may contribute to pathology in RA (and render RA patients resistant to future treatment with HDAC inhibitors) [2]. Kawabata and colleagues, in contrast, argue that increased HDAC1 activity may contribute to RA and represent a new therapeutic target [1].

The studies conducted by both groups are technically impeccable, ruling out many trivial explanations for discordant results. However, Kawabata and colleagues noted that none of the patients they examined was treated with TNF $\alpha$-blocking biologicals and that synovial TNF $\alpha$ production significantly correlated with HDAC activity and HDAC1 expression. In contrast, a substantial 
number of the RA patients studied by Huber and colleagues [2] received TNF $\alpha$-blocking therapies, raising the possibility that TNF $\alpha$ drives HDAC expression and activity. Although independent analyses of larger patient cohorts and prospective clinical studies are needed to substantiate this idea in vivo, initial in vitro experiments showing that TNFo stimulation induces RA FLS HDAC activity and HDAC1 expression [1] are compelling.

Several aspects of these two studies deserve further exploration. Variation in HDAC activity and expression between RA and non-inflammatory OA observed by both groups is modest, and little is known about the magnitude of fluctuation in cellular HDAC activity sufficient to modify inflammatory responses. Analyses of mice expressing only single alleles of specific HDACs in experimental arthritis models may be useful in answering this question. It is also uncertain whether altered synovial HDAC activity influences therapeutic strategies targeting the balance of synovial protein acetylation, as HDAC inhibitors are uniformly effective in animal models of RA [5] and demonstrate anti-inflammatory properties in RA FLSs [6], synovial macrophages, and synovial biopsy explants [7]. Kawabata and colleagues also provide evidence strengthening the hypothesis that HDACs are intimately involved in inflammatory signal transduction pathways in RA. The timing of changes in HDAC activity and expression following TNF $\alpha$ stimulation corresponds with the involvement of signaling proteins required for FLS cytokine responses, including nuclear factor-kappa-B (NF-kB) p65, JAK/STAT (Janus kinase/signal transducer and activator of transcription) signaling components, AP-1 (activator protein-1), and p53. These proteins are regulated by reversible acetylation [5], and the biochemical effects of acetylation (regulation of protein activation, localization, stability, and target specificity) are as diverse as those regulated by phosphorylation [8]. Indeed, recent evidence has demonstrated that, in transformed RA FLSs, HDAC inhibitors prevent activation of NF-кB p65 [9].

Finally, while HDAC1 is the most prominent Class I/II HDAC in RA synovial tissue and FLS $[1,6]$, silencing of either HDAC1 or HDAC2 have similar effects on FLS survival and proliferation [6]. This may suggest either that there are numerous redundancies in HDAC target specificity, or that bulk HDAC activity is most relevant to inflammatory and pro-survival gene expression in RA, important in considering whether inhibition of specific HDACs will be needed in therapeutic strategies. Reaching a consensus on the expression and activity of new biological targets in RA is a critical step in the evaluation of their role and therapeutic potential. However, as exemplified by the burst of recent research on HDAC biology in RA, the initial disparate results and conclusions are what accelerate our drive to spark the most intriguing questions and reach this consensus.

\section{Abbreviations}

COPD, chronic obstructive pulmonary disease; FLS, fibroblast-like synoviocyte; HAT, histone acetyltransferase; HDAC, histone deacetylase; NF-kB, nuclear factor-kappa-B; OA, osteoarthritis; RA, rheumatoid arthritis; TNFa, tumor necrosis factor-alpha.

\section{Competing interests}

The authors declare that they have no competing interests.

Published: 11 October 2010

\section{References}

1. Kawabata T, Nishida K, Takasugi K, Ogawa H, Sada K, Kadota Y, Inagaki J, Hirohata S, Ninomiya Y, Makino H: Increased activity and expression of histone deacetylase 1 in relation to tumor necrosis factor-alpha in synovial tissue of rheumatoid arthritis. Arthritis Res Ther 2010, 12:R133.

2. Huber LC, Brock M, Hemmatazad H, Giger OT, Moritz F, Trenkmann M, Distler JH, Gay RE, Kolling C, Moch H, Michel BA, Gay S, Distler O, Jüngel A: Histone deacetylase/acetylase activity in total synovial tissue derived from rheumatoid arthritis and osteoarthritis patients. Arthritis Rheum 2007, 56:1087-1093.

3. Adcock IM, Tsaprouni L, Bhavsar P, Ito K: Epigenetic regulation of airway inflammation. Curr Opin Immunol 2007, 19:694-700.

4. Strietholt S, Maurer B, Peters MA, Pap T, Gay S: Epigenetic modifications in rheumatoid arthritis. Arthritis Res Ther 2008, 10:219.

5. Grabiec AM, Tak PP, Reedquist KA: Targeting histone deacetylase activity in rheumatoid arthritis and asthma as prototypes of inflammatory disease: should we keep our HATs on? Arthritis Res Ther 2008, 10:226.

6. Horiuchi M, Morinobu A, Chin T, Sakai Y, Kurosaka M, Kumagai S: Expression and function of histone deacetylases in rheumatoid arthritis synovial fibroblasts. J Rheumatol 2009, 36:1580-1589.

7. Grabiec AM, Krausz S, de Jager W, Burakowski T, Groot D, Sanders ME, Prakken BJ, Maslinski W, Eldering E, Tak PP, Reedquist KA: Histone deacetylase inhibitors suppress inflammatory activation of rheumatoid arthritis patient synovial macrophages and tissue. J Immuno/ 2010, 184:2718-2728.

8. Glozak MA, Sengupta N, Zhang X, Seto E: Acetylation and deacetylation of non-histone proteins. Gene 2005, 363:15-23.

9. Choo QY, Ho PC, Tanaka Y, Lin HS: Histone deacetylase inhibitors MS-275 and SAHA induced growth arrest and suppressed lipopolysaccharidestimulated NF-kappaB p65 nuclear accumulation in human rheumatoid arthritis synovial fibroblastic E11 cells. Rheumatology (Oxford) 2010, 49:1447-1460

doi:10.1186/ar3137

Cite this article as: Grabiec AM, Reedquist KA: Histone deacetylases in RA: epigenetics and epiphenomena. Arthritis Research \& Therapy 2010, 12:142. 